\title{
Smart Healthcare Approach using Big Data: Big Opportunities and Challenges
}

\author{
Jagjit kaur*, Purnima Popli \\ Department of Computer Science and Engineering \\ CGC College of Engineering, Landran, Mohali, India \\ Email Id:* jagjitk.coecse@cgc.edu.in
}

\begin{abstract}
Big data' is defined as the collection of large amount of data in digital form irrespective of the fact that it is whether structured or not. In this modern era, vast amount of data is created everywhere every day at exponential rates. The primary objective of this paper is to provide deep analysis in the area of healthcare industry using big data. There is vast possibility of advanced patient care and decision support for physical and clinical data. As hospitals stores records of various patients and by collecting and analyzing this clinical data available from various geographical locations, we can predicts the behavior analysis and symptoms of patients under critical conditions and later by analyzing this data we can improve our medication facilities and service centers. In this article, we have discussed the usage of big data in healthcare and corresponding decision support and how the processing steps can be carried out for providing benefits to society.
\end{abstract}

Index Terms - COVID-19,C T,DNA,personal health record(PHR), data evaluation (Keywords)

\section{INTRODUCTION}

Big data is widely used term now a days. It is Increasing day by day. Healthcare is one of the field in which improvement is needed. If we discuss more about big data, its upcoming technology, capability and its essence in health industry we will be surprised in future, hospitals will no longer be as crowded. Online examination, daily checkups, prescriptions everything will be provided online. Certain apps are also introduced like MedCare by then. In this paper we focus on the digitalis ation, uses, objectives and some process which are taken in notice for various operations in this field. Big data is expanding day by days like if we notice the current situation of the world which is caused by COVID-19. No businessman, entrepreneur, industrialists etc. Cannot do anything the cure of the disease is only in hands of the doctors. The researches, technology, digitalisation are the only key to get over it and behind this big data plays a crucial role. This will help the scientists to fight against any dis ease which comes over. These techniques are very us eful to solve problems. The storage of vast information is also done by big data. Though only one sector have been discussed in this paper, there are many other areas where big data is used and in future big data is going to be proved as a boon for researchers to overcome all the hurdles. All this also requires careful design of experiments and models, cooperation among experimentalists, computational scientists and clinicians[1]. That's why big data and analytics is a step by step procedure of research, discovery of new matter, analysing the data, understanding the data and the useful outcomes in future. The first and foremost key for anything to be started is information, the more information or we can say that more correct information can deliver best outcomes. That is why data collection plays a crucial role in every sector. In today's scenario we are flooded with lot of information of every aspects of life like science, technologies, social activities, health and many more. The technology is working upon how to gather, analyse, work and store the data. For that purpose the term 'big data' is launched to predict and analyse the unmanageable data, so WHAT IS BIG DATA?
Big data is nothing but a field that treats, extract information, deals with large data sets and process the required information.

\section{LITERATURE REVIEW}

In traditional way of storing data especially in handwritten form that is piling thousands of paper over paper, digitalization of this data (now call it Big data) would be of great to the hospitals, health care centres and research labs. Now when a new pharmaceutical form of drug is brought into practice as the medicine, it lacks the trial of the same over the people. You have seen wooden locked boxes in hospital with 'Drug Interactions 'written over it. Whenever any physician came across any particular type of hypersensitivity, contraindication or interaction with a particular medicine, he/she would be filling a form regarding the same and deposit it in the box with his findings. From the various hospital in the same way having several boxes hanging over there, data is collected and sent to the national centre for analys is. Now digitalization of that tremendous amount of data is done and sent to the world organizations like WHO, FDA and international pharmaceutical companies. Now this information is in zeta bytes[2]. From there these organizations manage the data and give new regulations for use of particular drug. As given in above example, Big Data is not only helpful but have become necessity.

Today electrical prescriptions are in trend. Why? Well for the differential diagnosis, doctor requires the history of past illness, any allergic reaction againstany medication, family history for differentiating congenital causes of presentation, any history of surgery orany blood transfusion history for the viral. Records of patient in electric form will not only save physician time for asking the same piece of stuff again and again but would be an ease for another doctor to continue the line of treatment in absence of other or shifting the patient from one department to another[3]. The prescription could be presented easily for any research or legal purposes. All the stuff- history of patients, lab finding, radiological findings in X-Rays, CTs etc- all in one place. Similarly hospital would be able to keep records of all the patients without 
any time limit or space limit for keeping the hardcopies easily.

\section{AIM OF BIG DATA IN HEALTHCARE SECTOR}

There are some objectives which were used to improve the field of healthcare. It offers convenient solution using big data which aims to investigation, abundance and approachability in the healthcare sector. Some proposed objectives are discussed below:-

Clinical Decision Support- The Clinical Decision Support (CDS) points to rise the standard of healthcare services by increasing the outcomes. It focuses on providing right information to the right people, proper healthcare management process, documentation and diagnosis. This will provide required information in any specific medical field.

> Disease Management- This system enables to examine various disease and perform certain tests. The analytics helps to improve the accuracy of finding information and also improves the outcomes. This demands the data support fromvarious sectors and organisations and from individual people.

$>$ Patient Matching- By using big data with the help of prescriptive analysis, patient-centred medical perspective develops. It focuses on previous details of diseases and management systems, symptoms of various diseases, curing methods etc. With the help of this it will become easy for doctors to study the patients reports and provide enough treatment to the patients after noticing the symptoms [8].

> Lifestyle Analysis-Lifestyle of a pers on plays a vital role in order to avoid medical dis orders and rise the preciseness towards disease dis covery. This will help us to examine the influence and causes of lifestyle activities done by individuals.

> Data Matching- Multiple medical organisations and many repositories are required to deal with it, In order to implement the system without any mistakes, preciseness and get greater results.

Data Security-In every aspect, It is always noticed that privacy is a foremost concern. As medical reports includes many private and personal information so it is allowed to limited people for verification and study. Data is stored with every required security and rules and regulations such as in health insurance portability.

$>$ Data Pyramid- The pyramid is us efulbecause it portray the architecture and explains about data management. It gives the clear picture of data flow from starting with raw data and its uses and types followed by the useful information and finally the goal of a system is achieved.

> Data Cycle-Data cycle includes various steps like data collection, processing, reduction and transformation, analysis and output. In the following section we will discuss how data is collected and perform steps and how some useful information is attained as an output[9].

\section{HOW BIG DATA IS USEFUL IN HEALTH SECTOR}

Healthcare is a multidimensional system whose aim is to prevent, diagnose and treat heath related issues in human beings. Healthcare comprises of health professionals (doctors or nurses), health facilities (hospitals, clinics) and the financing companies to run the institution for above cause. Healthcare is needed in various heath sectors like medicine, dentistry, nursing, psychology, physiotherapy and many others. Healthcare professionals are also of many types like surgeon, dentists, scientists, Cardiologists, dermatologists, endocrinologists, neurologists, psychiatrists etc. At all these levels, these doctors need a medical history of patients, medical data and other required confidential history for the treatment[4].

So to gather all this required information paper file reports were in use earlier but now a day's 'electronic health records, (EHR) is in practice. The advent of computer and technology made this possible to represent, transfer, experience, gather, join and control data for providing services in the healthcare field.

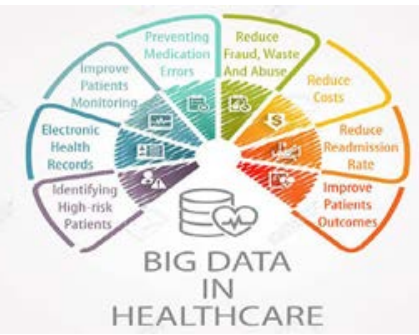

Fig. 1. Big Data Healthcare system

\section{A. Digitalisation}

Like EHR, electronic medical record (EMR) and personal health record (PHR) and also other one medical practice management software (MPM), despite these there are many more healthcare data components which are used to gather the information of patients to improve the quality, service efficiency and costs of healthcare along with reduced medical errors.

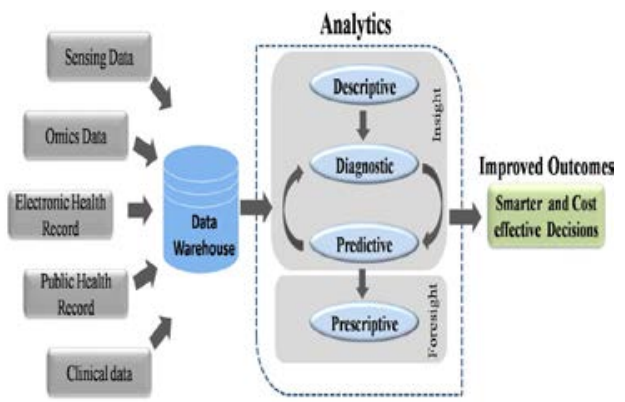

Fig. 2. Analytic behaviour in Healthcare system

The big data in healthcare focuses on controlling costs and improves health. The data is expanded among many healthcare systems for detailed investigation on it and all the information remains confidential. After many decades today's technology make it happen to collecthuge amount 
of information about diseases also each and every detailed data of patients and the data of patients which get wasted some of the time. This is the Workflow of Big data Analytics. The Data warehouses gather vast amounts of data produced through various sources. This data is performed by using analytic pipelines to acquire correct and modest healthcare options.

The Big data makes the world, better place to live with digitalised innovations in various aspects specially in healthcare. It enables us to deal with various problems related with healthcare like gathering fitness data, improve preventive care, discovering new drugs and various treatment to cure diseases.

\section{B. Corona Virus- Today's Pandemic!}

SARS Corona Virus-2 is known to cause the COVID19 dis ease has appeared to shut down the world for a while. In this period Big data is required by health profession to collect the data fromdifferent institutes and integrate the same at one place. WHO is currently maintaining the data of every patient mean while the res earches continued. For the purpose different departments of different research centres are working continuously. For the same data from different departments are collected by Medicine department to work out the line of treatment. Biochemistry department works on the strain difference that the CORONA has acquired, Microbiology department collects the data fromsame and brings the structural analys is and virulence factors of the same. Now all the data is maintained at singleplace and laboratory test techniques are developed from the same. Now pathology department works in collaboration tobring about thepathogenesis and laboratory findings too. Meanwhile department of Pharmacology works on therapeutic drugs and bring about the same. In the meanwhile 'Department of Preventive And Social Medicine' has worked enough to find themode of transmission of virus and bring about the idea of 'social distancing.' Now the clinical departments come in the game. All the information got from the above is analyzed by Department of Medicine and a set line of treatment is established[5]. The efficacy of the same line of treatment is observed by collecting information of different patients and altered ways are too established. Meanwhile 'Department of gynaecology and 'paediatrics' for line of treatment in pregnant, lactating woman and children. Radiological findings are established to correlate with the clinical findings.

Now all the above research lines contain data and only data. Not only data is to be conjured but instead have to be kept and analyzed by different institutes and departments for further researches. Thus manipulation, integration, analysis, regular update of data could not be done in traditional way. Thus Big Data is must.

\section{PROCESSING OF BIG DATA IN HEALTH CARE SYSTEM}

\section{A. Health Tracking}

Big data and analysis enables us to track patient's sleep, heart rate , exercise, distance walked, calories required etc. Also many more technology is introduced which can measure the Hb level, blood pressure etc. The uninterrupted track of body es sentials along with other data gives permission to healthcare centre to avoid incoming of people to the hospitals and also provide necessary care before their condition goes inadequate level.

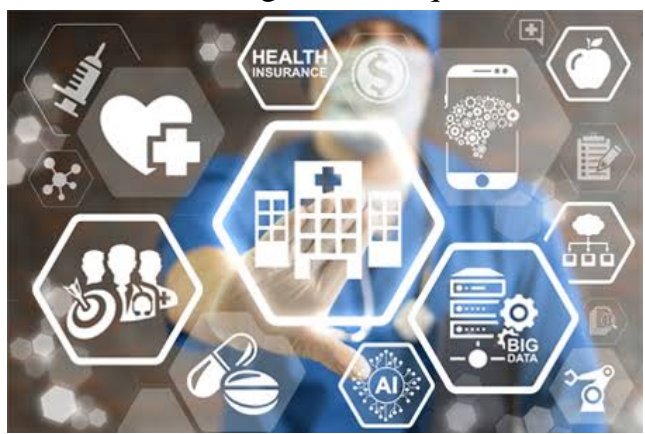

Fig.3. Health tracking system

\section{B. Minimize Cost}

Big data can be a best way to minimise the costs for hospitals. With the help of some resources we can easily predict the no of patients who are coming to the hospitals daily and this will reduce the requirement of staff members and that money will also be invested in some other resources like equipments and all. With the help of technology patients can check their reports online and view other necessary details like prescription of medicine, OnOff of hospitals, timings, costs etc this is helpful for patients as they have to spend less time in hospitals and also the crowd in hospital will be maintained[6]. All this will automatically reduce costs and with the help of some people the hospital will run smoothly.

\section{Collaborating High-Risk Patients}

If all the data of patients is uploaded online it will be a easy check on the details and history of patients. Doctors can easily study the previous and current reports of their patients and there will be no paper work which has to be kept safely, the online data is safe there. These details of patients gives the doctor perfect idea of check-ups, incoming of patients, prescription, acute and chronic dis eas es and will help the patients by providing proper care and nurturing this is a great way of check-ups with proper care of high- risk patients.

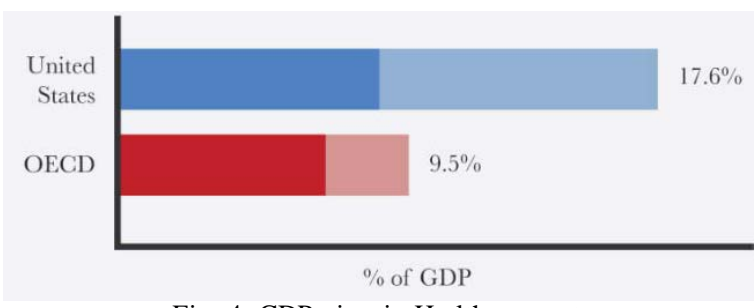

Fig. 4. GDP view in Healthcare system

\section{Anticipating Human Errors}

As noticed in many cases that professionals fails to understand the cause of disease so they prescribe wrong medicines and also wrong treatment to the patients these errors cambe corrected by big data it can analyse the data and reduce mistakes and save lives. This can be us eful to doctors also as they cater many patients in a day.

\section{E. Advancement in Healthcare Sector}

Big data proves to be a boon for development in science and technology like in healthcare, machine learning, an artificial intelligence etc can be used to surf 
the data in millisecond and provides the solutions for various diseases. This will become more advanced by the collection of more data and it will provide accurate and unique solutions. Predictivestudy of patients will help the doctor to study many patients of same disease and also prevent the travelling of patients from one place to another[7].

Hence, Big Data enhances the ability of the healthcare sectors to:

- $\quad$ Predict Epidemics

- $\quad$ Cure Disease

- Improve Quality of Life

- Increase Preventable Care

- Begin Early Preventive Care

\section{ANALYZING AND EVALUATION OF DATA}

A. Data Collection: Collection is the first and foremost step of every procedure it includes collection of information from various sources, managing and storing it. Data is of two types like structured and unstructured. It includes clinical reports, patients history, medical requirements, health index, social content etc.

B. Data Pre-processing: After collection of data from various inputs and storing it to a particular storage we moves further towards processing stage. In this stage it clears the data, sort it accordingly and find all the missing values and remove the junk data. Preparing of data plays a vital role in shifting the process ahead. The processed data is useful to achieveany results not a raw material. So it is an important process.

C. Data Reduction \& Transformation: This stage helps us to avoid less important data. Many operations like mathematical and compression algorithms are used to transform the data into useful data. It will not lose any information but helps to simply the process smoothly.

D. Data Analytics: Data analytics is another important part of processing as it gives conclusions after analysing the data. It translates the data for experimentation, science and some business outcomes. Using these conclusions we can achieve higher, greater goals .

E. Data Output: The final report is given on the basis of inputs we have. The report is fully examined in various formats in order to provide the user proper view to monitor or print. The output data is worthwhile and correct information is provided to achieve greater goals and for this output we need a storage medium to store that information. It is very important to maintain the security system, morality and control on access of data throughout the life cycle process[10].

\section{ROLE OF STATISTICS IN HEALTHCARE FIELD}

The practice of collecting and analysing big data in any field is called statistics and it is important in every field but particularly in healthcare there are statistics which shows us what is the expenditure on healthcare, how many people are under insurance of heath, how many diseases are there, pattern of disease in population, vaccines introduced and many more other statistics of data. How much incentives are spend on healthcare and percentage is shown as an example in following data it focuses on medicines prescription, healthcare services and doctors services.

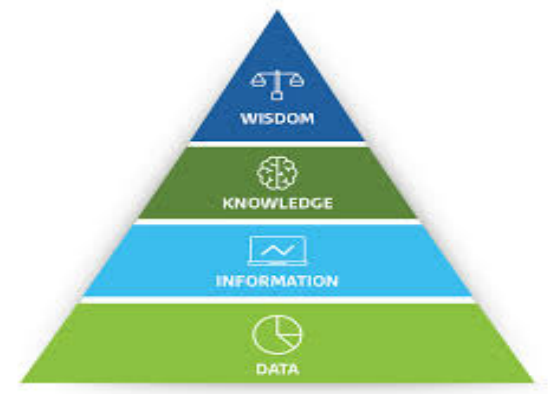

Fig.5. Big Data evaluation chart

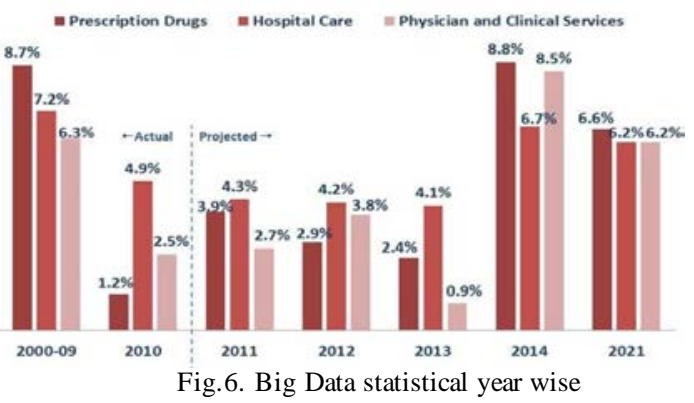

\section{FUTURE SCOPE OF HEALTHCARE SYSTEM USING BIG DATA}

1. The AI will revolutionise diagnostic practices.

2. Alexa will be able to diagnose cold.

3. You will be able to get medicines at home without visiting a doctor.

4. With the help of drones you were able to give your blood samples to doctors for those people who live in rural areas[11].

5. Healthcare insurance will include more incentives which were provided to patients to improve their health.

\section{CONCLUSION}

In this paper we have discussed about big data, its emerging technology, efficiency and its usage which clear the significance of healthcare industry. In current scenario data is getting generated at a dramatic pace. So it become a challenge to analyze and recognise this emerging data which is tough task to general man. To from this survey, we come to this conclusion that every big data platform has its individual focus and specific functional programs. IF big data is used in healthcare industry it can have magical effects in health sector at real-time analytic. Different methods, benefits, current situation pandemic issues discussed.

\section{REFERENCES}

[1]. A. Gandomi and M. Haider, Beyond the hype: Big data concepts, methods, and analytics, International Journal of Information Management, 35(2)(2017), pp.137-144.

[2]. M. K.Kakhani, S. Kakhani and S. R.Biradar, Research issues in big data analytics, International Journal of Application or Innovation in Engineering \& Management, 2(8) (2018), pp.228-232. 
[3]. A. Gandomi and M. Haider, Beyond the hype: Big data concepts, methods, and analytics, International Journal of Information Management, 35(2)(2017), pp.137-144.

[4]. C. Lynch, Big data: How do your data grow?, Nature, 455 (2008), pp.28-29.

[5]. X. Jin, B. W.Wah, X. Cheng and Y. Wang, Significance and challenges of big data research, Big Data Research, 2(2) (2015), pp.59-64.

[6]. R. Kitchin, Big Data, new epistemologies and paradigm shifts, Big Data Society, 1(1) (2014), pp.1-12.

[7]. C. L. Philip, Q. Chen and C. Y. Zhang, Data-intensive applications, challenges, techniques and technologies: A survey on big data, Information Sciences, 275 (2014), pp.314-347.

[8]. K. Kambatla, G. Kollias, V. Kumar and A. Gram, Trends in big data analytics, Journal of Parallel and Distributed Computing, 74(7) (2014), pp.2561-2573.

[9]. S. Del. Rio, V. Lopez, J. M. Bentez and F. Herrera, On the use of mapreduce for imbalanced big data using random forest, Information Sciences, 285 (2014), pp.112-137.

[10].MH. Kuo, T. Sahama, A. W. Kushniruk, E. M. Borycki and D. K. Grunwell, Health big data analytics: current perspectives, challenges and potential solutions, International Journal of Big Data Intelligence, 1 (2014), pp.114-126.

[11].R. Nambiar, A. Sethi, R. Bhardwaj and R. Vargheese, A look at challenges and opportunities of big data analytics in healthcare, IEEE International Conference on Big Data, 2019, pp.17-22.

[12].T. K. Das and P. M. Kumar, Big data analytics: A framework for unstructured data analysis, International Journal of Engineering and Technology, 5(1) (2016), pp.153-156.

[13].T. K. Das, D. P. Acharjya and M. R. Patra, Opinion mining about a product by analyzing public tweets in twitter, International Conference on Computer Communication and Informatics, 2014.

[14].L. A. Zadeh, Fuzzy sets, Information and Control, 8 (1965), pp.338- 353. [15] Z. Pawlak, Rough sets, International Journal of Computer Information Science, 11 (1982), pp.341-356.

[15].D. Molodtsov, Soft set theory first results, Computers and Mathematics with Aplications, 37(4/5) (1999), pp.19-31.

[16].J. F.Peters, Near sets. General theory about nearness of objects, Applied Mathematical Sciences, 1(53) (2007), pp.2609-2629.

[17].R. Wille, Formal concept analysis as mathematical theory of concept and concept hierarchies, Lecture Notes in Artificial Intelligence, 3626 (2005), pp.1-33. 